\title{
Impurity Breakdown in GaAs Samples Grown by Molecular Beam Epitaxy
}

\author{
R.M. Rubinger, A.G. de Oliveira, G.M. Ribeiro, J.C. Bezerra, C.M. Silva, \\ W.N. Rodrigues, and M.V.B. Moreira \\ Departamento de Física, Universidade Federal de Minas Gerais \\ CP702, 30123-970 Belo Horizonte, Brazil
}

Received February, 1999

\begin{abstract}
In this work we present studies for the behavior of impurity breakdown of two GaAs samples grown by Molecular Beam Epitaxy at $200^{\circ} \mathrm{C}$ and $300^{\circ} \mathrm{C}$. We vary the temperature and the illumination intensity. For the sample grown at $200^{\circ} \mathrm{C}$, the transport mechanism after the breakdown is of the same type of the main free carriers at low electric fields. Below $100 \mathrm{~K}$, a clear dependence of the threshold electric field with temperature was observed. The sample grown at $300^{\circ} \mathrm{C}$ shows a breakdown due to an acceptor level at $41 \mathrm{meV}$ from the valence band. The threshold electric field increases with illumination due to the generation of free electrons by deep levels. A similar behavior was also observed for a Cr doped GaAs sample.
\end{abstract}

\section{Introduction}

Negative differential conductivity (NDC) has been studied since the early 60 's. Basically it was studied with the intention to develop microwave devices for engineering applications. In the 80 's, studies of nonlinear dynamics and chaotic phenomena have started the development of a new class of semiconductor devices where nanostructures details should play important roles. We have studied the current-voltage $(\mathrm{IxV})$ characteristics of GaAs samples grown by Molecular Beam Epitaxy (MBE) at low temperatures (LT). We have compared the results of two samples, one grown at $200^{\circ} \mathrm{C}$ (sample $\mathrm{BH} 9801$ ) and other at $300^{\circ} \mathrm{C}$ (sample BH9525). These results were also compared with those obtained from a standard commercial Cr-doped GaAs substrate. For the semi-insulating (SI) GaAs:Cr sample a $\mathrm{Cr}^{3+}$ charge state accepts electrons from shallow donors becoming a $\mathrm{Cr}^{2+}$. We were interested in studying the behavior of the impurity breakdown $[1,2]$ in LTMBE GaAs samples due to the presence of a high density of intrinsic deep traps, mainly in the form of antisite defects, namely, As atoms in the Ga site $\left(\mathrm{As}_{G a}\right)$. These intrinsic defects give the samples the SI characteristic since the Fermi level is mid-gap positioned. Under a high electrical field the residual free electrons in the conduction band for SI GaAs:Cr samples and free holes in the valence band for LT-MBE GaAs samples are accelerated. At certain values of the electric field these hot carriers, when colliding with impurities, have enough energy to release further carriers. This is the so-called impact ionization process and it has a strong nonlinear dependence on the electric field and on the carrier density. In the present work we have studied the temperature dependence of the $\mathbf{j x} \mathbf{E}$ characteristics in darkness and under different illumination intensity. We have used an infrared LED as the light source. Hall measurements were also carried out in order to obtain parameters necessary in order to calculate the activation energy and the ionization cross sections of the impact ionization process. The nonlinear regime of the jxE characteristics permits the assessment of important parameters like the activation energy of deep traps, their densities, and their ionization cross sections. For the onset of instabilities, electric fields of the order of a few thousand of $\mathrm{Vcm}^{-1}$ are necessary. For this work, typical samples were cut into small rectangles and two indium contacts were defined and separated by about $200 \mu \mathrm{m}$. LT-MBE GaAs samples are photo-sensitive and for this reason measurements under illumination were carried out. We have observed that the density of intrinsic defects increases with the reduction of the growth temperature, in agreement with data of the literature [3]. The low resistivity value at room temperature for the sample grown at $200^{\circ} \mathrm{C}$ suggests a higher density of shallow defects. Consequently, some tentatives carried out in order to observe the instabilities at room temperature have resulted in the sample destruction and for the observation of the nonlinear regime a temperature reduction was required. 
We have observed a rich phenomenology. The more important effects are those in association with the ionization of deep traps by hot carriers. As a function of temperature and illumination intensity we have observed the presence of strong negative differential conductivity (NDC) regions having both $\mathrm{S}$ and $\mathrm{N}$ shapes. Based on the value of the activation energy we have concluded that different deep traps are involved in each of the three samples. It means that the intrinsic characteristics of the deep defects must change drastically between $200^{\circ} \mathrm{C}$ to $300^{\circ} \mathrm{C}$. The study of the temperature dependence for the sample grown at $300^{\circ} \mathrm{C}$ suggests the presence of at least two different deep traps which were associated with two different slopes in the nonlinear part of the $\mathbf{j x E}$ characteristics after two observed $\mathrm{S}$ instabilities. At the highest values of the used electrical field the observed $\mathrm{N}$ shaped NDC region was associated with inter-valley repopulation.

\section{Results}

\section{II.1 Sample BH9801}

The temperature dependence of the $\mathbf{j} \mathbf{x} \mathbf{E}$ characteristic in darkness is show in Figs. 1 and 2. The threshold field is around $3000,2550,2850,2550$, and $3000 \mathrm{Vcm}^{-1}$ at $125,150,175,200$, and $225 \mathrm{~K}$, respectively. These fluctuations in the threshold field value are related to the change in the free carrier density. The fluctuations are controlled by the Fermi distribution of the defect involved with the impact ionization process and the compensation character of the deep levels, which is a function of its carrier lifetime. We have also observed that the slope of $\mathbf{j x E}$ curve in the impact ionization regime reduces for decreasing temperature. For temperatures below about $150 \mathrm{~K}$ the $\mathbf{j x E}$ curve bends downward even in the non-linear regime. In this situation even a NNDC behavior could be observed.

For the results shown in Fig. 2 the intensity of the jump in the current density at the breakdown is significantly stronger than those observed at higher temperatures and the value of the threshold field decreases monotonically for increasing temperatures. The threshold fields are 8600 and $5000 \mathrm{Vcm}^{-1}$ at 25 and $100 \mathrm{~K}$, respectively. In this temperature range the carrier lifetime for the decay into the deep levels of the $\mathrm{As}_{G}$ a defect is longer than the band to band recombination time. Therefore the impact ionization process depends basically on the free carriers provided by the Fermi distribution of carriers from the deep trap involved in the impact ionization process. Another characteristic of these measurements is that the current after the threshold shows complex behaviors.

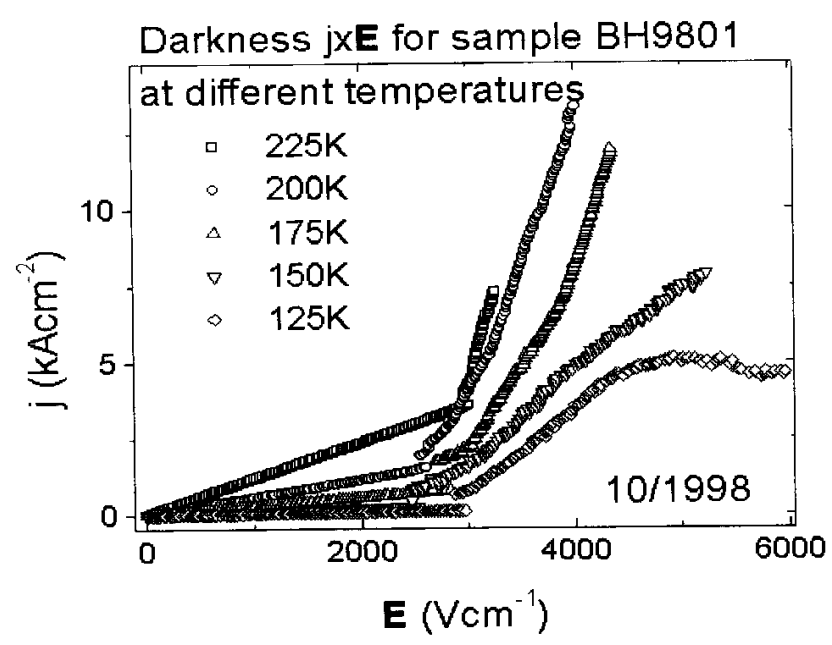

Figure 1. Two effects can be observed in these $\mathbf{j x E}$ characteristics: a) the behavior of the threshold, which fluctuates for $\mathrm{T}$ in the range $300-125 \mathrm{~K}$; b) the form of the current density. For temperatures below $125 \mathrm{~K}$ see fig. 2 .

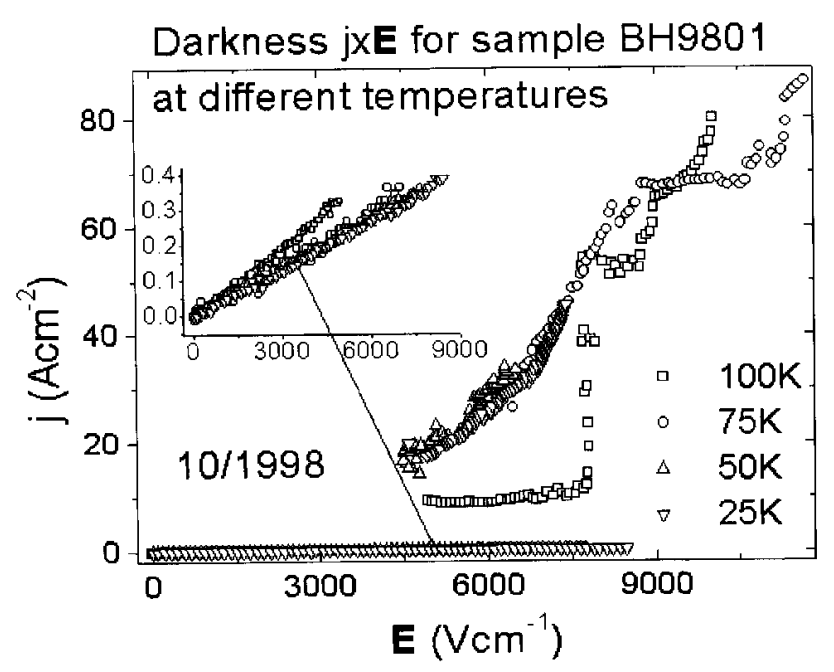

Figure 2. The slope of the $\mathbf{j x} \mathbf{E}$ characteristics changes drastically after the threshold and it decreases with the temperature as can be seen in the inset. The current density after the threshold shows complex behavior presenting low frequency oscillations, NNDC region and many changes in the slope.

\section{2 Sample BH9525}

The temperature dependence of the $\mathbf{j} \mathbf{x} \mathbf{E}$ characteristic in darkness is show in Fig. 3. For temperatures bellow $225 \mathrm{~K}$ the threshold was not observed at least for electric fields up to around $6000 \mathrm{Vcm}^{-1}$. Otherwise, above $225 \mathrm{~K}$ the threshold is clearly observed and there is no significant change in the slope. However low frequency oscillations (LFO) which are observed over the whole range of the applied electric field disturbs the curve and the precise location of the first threshold (around $2500 \mathrm{Vcm}^{-1}$ ) could not be determined. For 
measurements at $296 \mathrm{~K}$ a second threshold could be observed around $3000 \mathrm{Vcm}^{-1}$. Fig. 4 shows the $\mathbf{j x E}$ characteristics at $296 \mathrm{~K}$ as a function of the illumination intensity. The value of the threshold field increases with the light intensity. In the range from $200 \mathrm{~K}$ to $300 \mathrm{~K}$ the slope of the curves after the threshold does not change with illumination but the jump intensity in the current density does. Under illumination the current density shows an ohmic regime only for very low electric fields and it changes to a sublinear regime that is preserved up to the threshold.

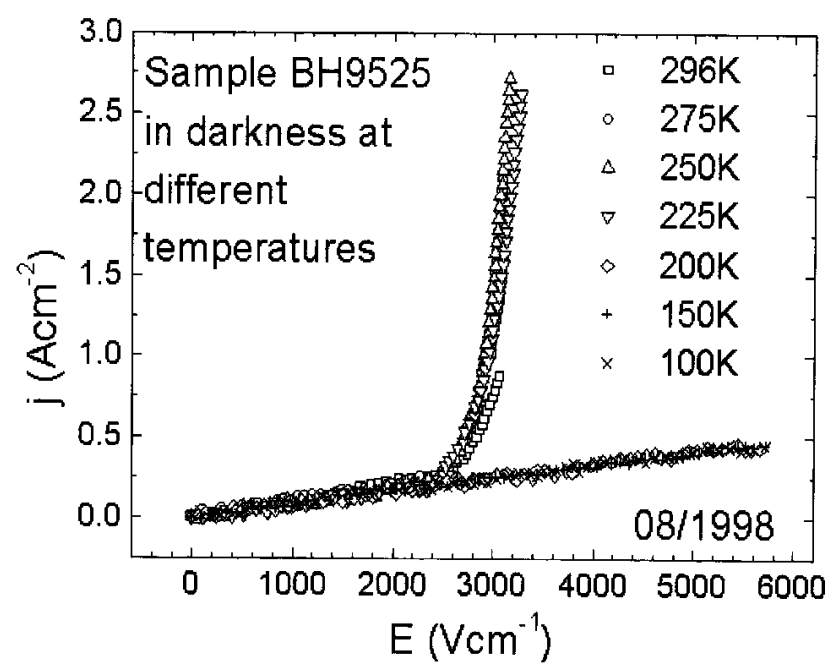

Figure 3. Until $6000 \mathrm{Vcm}^{-1}$, no threshold could be observed bellow $225 \mathrm{~K}$ in the $\mathbf{j x E}$ curve. At $296 \mathrm{~K}$ a second threshold can be observed around $3000 \mathrm{Vcm}^{-1}$.

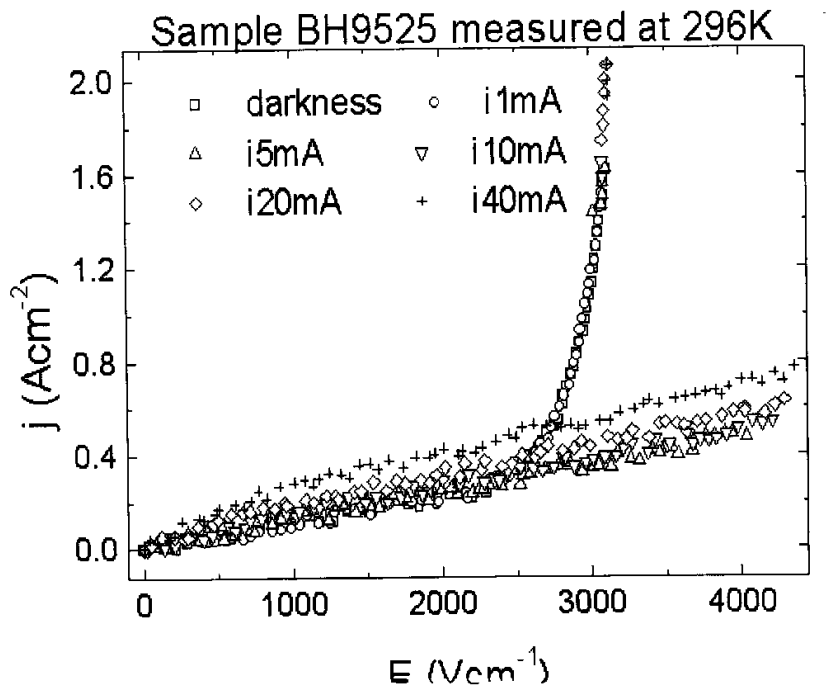

Figure 4. The threshold and the jump in the current density increase with the light intensity but the curve fits each other after the threshold.

In Fig. 5 we show the jxE characteristics under illumination at $225 \mathrm{~K}$. The value of the threshold field increases with the light intensity in the same way as observed at $296 \mathrm{~K}$. Two thresholds could be observed. The slope after the first threshold is smaller than that after the second one, which suggests impact ionization in two different defects. The slope after the second seems to be same as observed at $296 \mathrm{~K}$. The sublinear behavior occurs for electric fields higher than about $500 \mathrm{Vcm}^{-1}$ and we claim that it is associated with field enhanced trapping. This assumption is corroborated by the presence of LFO.

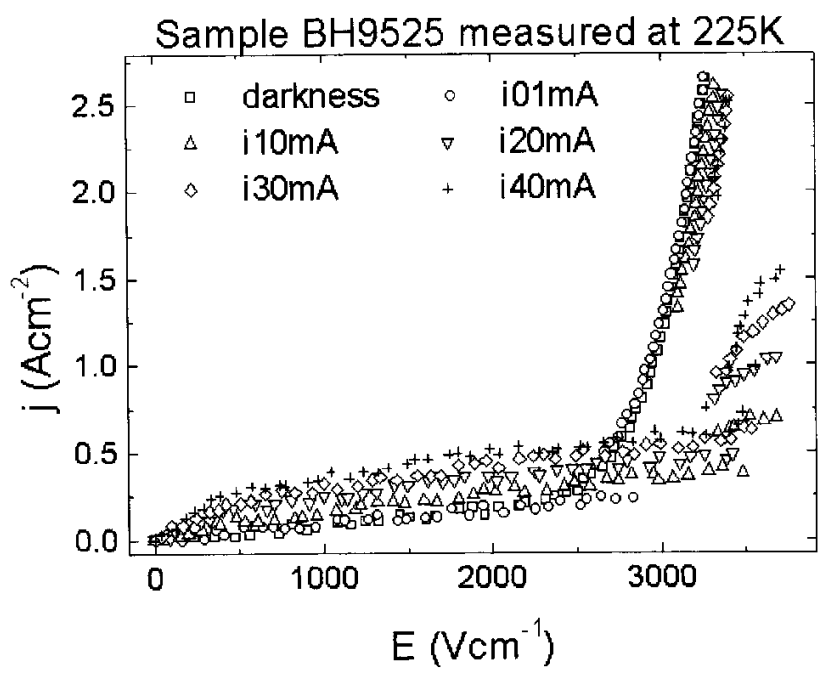

Figure 5. At $225 \mathrm{~K}$ and below two thresholds were observed. The first has a smaller slope than the second. The slope of the current density after the second threshold seems to be related with the same phenomena as that observed at $296 \mathrm{~K}$. Above $500 \mathrm{Vcm}^{-1}$, a sublinear behavior is observed which is probably related to field enhanced trapping.

\section{Discussion}

We have observed impurity breakdowns, which switches between the low and high current density for both samples, with a threshold field around $2500 \mathrm{Vcm}^{-1}$ at $225 \mathrm{~K}$. This threshold is too low to be related with the threshold switching of an EL2-like center (which is expected to occurs at around $8 \mathrm{kVcm}^{-1}$ ). Because of the LT growth our samples should present a high density of such defects. Therefore the threshold we are observing may be related to other traps. The increase in the value of the threshold field with illumination for sample BH9525 suggests that impact ionization generates a different type of carrier rather than the one who determines the transport mechanism at low fields. Indeed we know that the EL2-like or $\mathrm{As}_{G a}$ deep levels releases carriers preferably in the conduction band for the infrared light we are using. In darkness we found a Hall mobility and a Hall p-type carrier density of $560 \mathrm{~cm}^{2} / \mathrm{V}$.s and $4.7 \times 10^{11} \mathrm{~cm}^{-3}$, respectively. 
In contrast, for sample BH9801 we have observed a decrease in the threshold field with illumination intensity. This suggests an enhancement in the carrier density under illumination involved in the impact ionization. For this sample the presence of strong LFO disturbs the Hall measurements and it was not possible to obtain reliable values for the mobility and carrier density.

Using the same procedure of other authors [2] we have assessed the energy associated with the impact ionization. For sample BH9525 we found $\mathrm{m}=560 \mathrm{~cm}^{2} /$ V.s; $1=2 \times 10^{-6} \mathrm{~cm} ; \mathrm{e}=41 \mathrm{meV} . \mathrm{l}$ is the mean free path and $e$ is the transition energy. These values are more compatible with holes then with electrons. At this point one might ask if $300 \mathrm{~K}$ is not a too high temperature for the existence of not ionized acceptors in a density high enough in order to induce impact ionization. We have calculated the Fermi distribution of holes due to this level and found $21 \%$ at $300 \mathrm{~K} ; 9.5 \%$ at $200 \mathrm{~K} ; 0.9 \%$ at $100 \mathrm{~K}$; and $10^{-19} \%$ at $10 \mathrm{~K}$.

Comparing the results with those of a GaAs:Cr sample, having a mobility of $4000 \mathrm{~cm}^{2} / \mathrm{V} . \mathrm{s}, \mathrm{p} 1 \mathrm{x} 10^{7} \mathrm{~cm}^{-3}$, and a mean free path of $1=5.5 \times 10^{-6} \mathrm{~cm}$, we found $\mathrm{e}=87 \mathrm{meV}$. Such an energy of $87 \mathrm{meV}$ is responsible for the release, through impact ionization, of electrons in the conduction band. Otherwise the effect of light is to release holes in the valence band. Thus, we claim that the same mechanism is responsible for the increase in the threshold field observed for sample BH9525.

In summary, we have measured the impurity breakdown of two LT-MBE GaAs samples at variable temperature and illumination conditions. Sample BH9801 shows a breakdown of the same type of the main free carriers and below $100 \mathrm{~K}$ shows a clear dependence of the threshold on the temperature. Sample BH9525, shows a breakdown of an acceptor level at $41 \mathrm{meV}$ from the valence band which increases the threshold under illumination due to the generation of free electrons by the EL2-like deep levels. This behavior is also observed for a GaAs:Cr sample.

\section{References}

[1] J. Hirschinger, F. -F. Niedernostheide, W. Prettl, V. Novák, and H. Kostial, Phys. Stat. Sol. 204, 477 (1997).

[2] C. Paracchini and V. Dallacasa, Solid State Comm. 69, 49 (1989).

[3] K. Krambrock, M. Linde, J. M. Spaeth, D.C. Look, D. Bliss, and W. Walukiewicz, Semicond. Sci. Technol. 7, 1037 (1992). 